Article

\title{
Effects of Achyranthes bidentata Polysaccharides on Intestinal Morphology, Immune Response, and Gut Microbiome in Yellow Broiler Chickens Challenged with Escherichia coli K88
}

\author{
Zhuying Liu ${ }^{1,2}$, Xiaolong Wang ${ }^{1}$, Shuqi Ou ${ }^{1}$, Muhammed Adebayo Arowolo ${ }^{1}$, De-Xing Hou ${ }^{2}$ \\ and Jianhua $\mathrm{He}^{1, * \mathbb{D}}$ \\ 1 College of Animal Science and Technology, Hunan Agricultural University, Changsha 410128, Hunan, China; \\ liuzhuying@gmail.com (Z.L.); wangxiaolong1982@gmail.com (X.W.); 15574942599@163.com (S.O.); \\ mbayor88@gmail.com (M.A.A.) \\ 2 The United Graduate School of Agricultural Science, Kagoshima University, Kagoshima 890-0065, Japan; \\ hou@chem.agri.kagoshima-u.ac.jp \\ * Correspondence: jianhuahy@hunau.net; Tel.: +86-731-8467-3916; Fax: +86-731-8461-8728
}

Received: 15 October 2018; Accepted: 3 November 2018; Published: 7 November 2018

\begin{abstract}
The present study was conducted to investigate the effects of dietary Achyranthes bidentata polysaccharide (ABPS) supplementation on performance, immune response, intestinal mucosal morphology, and gut microbiome in yellow-feathered broilers challenged with Escherichia coli K88. A $2 \times 2$ factorial design was used for the trial. Two hundred and forty one-day-old female broilers were randomly assigned to four treatments: (1) negative-control broilers were fed by a basal diet and saline (NG); (2) positive-control broilers were fed by a basal diet and orally challenged with $10^{8} \mathrm{CFU}$ E. coli K88 (CNG); (3) ABP group broilers were fed by a basal diet containing ABPS (500 mg $/ \mathrm{kg}$ of feed) and saline; (4) CABP group broilers were fed by a basal diet containing ABPS (500 mg/ $\mathrm{kg}$ of feed) and orally challenged with $10^{8} \mathrm{CFU}$ E. coli K88. Growth performance, serum biochemical indexes, immune responses, intestinal mucosal morphology, and cecal microbial community structure were evaluated. The ABP group had greatest body weight (BW), average daily body weight gain (ADG), and intestinal villus height compared to other treatments $(p<0.05)$. The CABP group had a higher villus height/crypt depth ratio $(\mathrm{V} / \mathrm{C})$ compared with other treatments $(p<0.05)$. The expression levels of NF- $\mathrm{KB}$ were lower in the ABP group. The CNG group had higher Escherichia coli and Enterococcus contents in cecal samples compared to other treatments $(p<0.05)$. Serum glucose, uric acid, TNF- $\alpha$, and Secretory Immunoglobulin A (S-IgA) levels were higher in broilers challenged with E. coli $(p<0.001)$ than that with saline. Broilers challenged with E. coli had reduced taxa richness in the cecal samples. Sequencing of $16 \mathrm{~S}$ rRNA genes in cecal samples revealed that a lower proportion of Firmicutes and a higher proportion of Proteobacteria were detected in the broilers challenged with $E$. coli. Compared with the controls, dietary ABPS supplementation increased serum total protein, albumin, and S-IgA levels, but decreased serum glucose, uric acid, and TNF- $\alpha$ levels in broilers $(p<0.05)$. Diet supplemented with ABPS increased the Firmicutes/Bacteroidetes ratio and the abundance of Ruminococcaceae and Lachnospiraceae, and altered cecal microbiota community structure. These results suggest that ABPS can promote growth performance and improve intestinal morphology and microbiota community structure in broilers challenged with E. coli K88.
\end{abstract}

Keywords: Achyranthes bidentata polysaccharides; immune response; intestinal mucosal morphology; cecal microbiota; Escherichia coli K88 


\section{Introduction}

The principal pathogenic bacteria associated with poultry causing foodborne illnesses are Campylobacter, Salmonella, and Escherichia coli [1]. Escherichia coli can cause an enteric disease known as colibacillosis in chickens, which in turn results in a high mortality rate and huge economic loss in the poultry industry [2,3]. Although colibacillosis can be prevented or treated by the use of antibiotics, there exist controversies regarding the prophylactic use of antibiotics in livestock production throughout the world. Therefore, alternative strategies have been actively investigated to replace these synthetic antimicrobial agents. Many studies have been published about different additives' effects on broiler chicken performance and immune response when challenged with Escherichia coli K88, such as prebiotics, probiotic, short-chain fatty acids, and essential oils, but little is known about polysaccharide additives [4-7]. These additives promote growth performance by increasing the average daily feed intake and improving immune function and intestinal villi development, but there has been very little research on the effects on the intestinal microbial community structure and balance of the host $[8,9]$.

Polysaccharides extracted from traditional Chinese medicines have been regarded as one of the alternatives, and a growing number of studies have shown that the polysaccharides have beneficial effects in promoting the growth performance and health of livestock animals [10]. Achyranthes bidentata polysaccharides (ABPS) are polysaccharides extracted from the Chinese herb Achyranthes bidentata. Several in vitro and in vivo studies have revealed the immunostimulating and anti-inflammatory effects of ABPS [11,12]. ABPS was also shown to enhance immune responses, increase feed intake, and promote growth when incorporated into swine diets, but little research has been carried out with poultry $[13,14]$. In our laboratory, we have observed a salutary influence of ABPS supplementation on pigs [15-17]. Moreover, our preliminary data showed that ABPS supplemented at the rate of $500 \mathrm{mg} / \mathrm{kg}$ of diet increased lymphocyte proliferation and improved immunity in yellow broiler chickens [18]. Based on these results, the present study was designed to investigate the effects of ABPS supplemented at the $500 \mathrm{mg} / \mathrm{kg}$ level of inclusion on growth performance, immune function, and gut microbiome community structure in yellow-feathered broiler chickens challenged with Escherichia coli K88.

\section{Materials and Methods}

\subsection{Birds, Diets, and Experimental Design}

\subsubsection{Preparation of ABPS and E. coli K88}

ABPS was extracted and prepared according to the procedure described by Chen et al. [15]. The extract was comprised of D-mannose and D-glucose in a molar ratio of 8:1 (HPLC analysis, Shanghai Institute of Chemical Physics, Chinese Academy of Sciences, Shanghai, China), and the final product was $95 \%$ ABPS as measured by vitriol anthrone using anhydrous glucose as standard control. The relative molecular mass of the ABPS was about 1300-1400 D as determined by the gel filtration method. The final extract was used for this trial [19].

The E. coli K88 strain was originally obtained from the College of Animal Sciences and Technology, Hunan Agricultural University, Changsha, China. The strain was inoculated in Luria Bertani broth for $24 \mathrm{~h}$ and cultured at $37^{\circ} \mathrm{C}$ with shaking ( $120 \mathrm{rpm}$ ). To determine the appropriate incubation time, a growth curve was constructed to establish the density of $E$. coli $\mathrm{K} 88$ required to reach the target gavage dosage of approximately $2 \times 10^{8} \mathrm{CFU} / \mathrm{mL}$.

\subsubsection{Animals}

All the experimental procedures were approved by the Institutional Animal Care and Use Committee of Hunan Agricultural University. In total, 240 1-day old female yellow-feathered broiler chickens (average BW = 30.4 g) were obtained from Hunan Wenshi Poultry Industrial Development Company, China. They were inspected upon arrival to ensure all chicks were free from any deformity and early signs of disease. Healthy chicks were randomly assigned to 4 treatments (with 6 replicates of 
10 chicks each). Each cage had raised wire floors and contained a self-feeder and waterer. The room temperatures were adjusted to $32{ }^{\circ} \mathrm{C}$ in the first week, $30{ }^{\circ} \mathrm{C}$ in the second week, $28{ }^{\circ} \mathrm{C}$ in the third week, and then $25^{\circ} \mathrm{C}$ to the end of the study.

\subsubsection{Diets and Experimental Design}

The diets used in this study were formulated based on the nutrient requirements of yellow-feathered broiler chickens (China, NY/T 33-2014) and the NRC (1994) (Table 1). All groups were fed the basal diet without growth promoters nor anticoccidia for the first 14 days, but were vaccinated against coccidiosis at placement. A $2 \times 2$ factorial design was used for the current study. The treatments were as follows: the negative treatment group received a basal diet and saline (NG); the positive treatment group challenged by E. coli K88 received a basal diet (CNG); the third group received a basal diet supplemented with ABPS (500 $\mathrm{mg} / \mathrm{kg}$ of feed) and saline (ABP); and the fourth group challenged by E. coli K88 received a basal diet supplemented with ABPS ( $500 \mathrm{mg} / \mathrm{kg}$ of feed) (CABP). E. coli $\mathrm{K} 88$ at the concentration of $2 \times 10^{8} \mathrm{CFU} / \mathrm{mL}$ of sterile saline was administered to the challenged groups at days 14,15 , and 16 . This was achieved by dropping $0.5 \mathrm{~mL}$ of the solution at the back of the oral cavity with the aid of a sterile syringe attached to a polyethylene tube, whereas the unchallenged groups were gavaged only with $0.5 \mathrm{~mL}$ of sterile saline. The E. coli K88-challenged and the unchallenged groups were kept in two separated rooms to avoid cross-contamination. Traditionally, yellow broiler chickens are always divided into early phase (1-28 day) and late phase (29-56 day); the experimental on the effects of ABPS supplementation was focused on the early phase, thus the experiment lasted 28 days.

Table 1. Composition of the basal diet.

\begin{tabular}{cc}
\hline Item & Basal Diet (0 to 4 weeks) \\
\hline Ingredient, $\%$ & 56.65 \\
Ground yellow maize & 36 \\
Soybean meal & 3.0 \\
Soybean oil & 1.8 \\
Dicalcium phosphate & 1.0 \\
Limestone & 0.3 \\
NaCl & 0.1 \\
DL-Met ${ }^{3}$ & 0.15 \\
Choline chloride & 1.0 \\
Premix ${ }^{1}$ & \\
Nutrient level ${ }^{2}$ & 12.22 \\
ME ${ }^{3}, \mathrm{MJ} / \mathrm{kg}$ & 20.10 \\
CP ${ }^{3}, \%$ & 1.02 \\
Lys, $\%$ & 0.42 \\
Met, $\%$ & 0.32 \\
Cys, $\%$ & 1.11 \\
Ca, $\%$ & 0.54 \\
Available $\mathrm{P}, \%$ & \\
\hline
\end{tabular}

${ }^{1}$ Supplied, per kilogram of diet: $\mathrm{Cu}, 10 \mathrm{mg} ; \mathrm{Fe}, 90 \mathrm{mg}$; Mn, $90 \mathrm{mg}$; Zn, $50 \mathrm{mg}$; Se, $0.2 \mathrm{mg}$;, $0.4 \mathrm{mg}$; Co, $0.4 \mathrm{mg}$; vitamin A, $5000 \mathrm{IU}$; cholecalciferol, $500 \mathrm{IU}$; vitamin E, $10 \mathrm{IU}$; riboflavin, $6.0 \mathrm{mg}$; pantothenic acid, $12 \mathrm{mg}$; niacin, $35 \mathrm{mg}$; cobalamin, $10 \mu \mathrm{g}$; biotin, $0.8 \mathrm{mg}$; folic acid, $0.8 \mathrm{mg}$; thiamine, $1.5 \mathrm{mg}$; and pyridoxine, $1.5 \mathrm{mg}$. ${ }^{2}$ Based on the Nutrient Requirements of yellow broilers (China, NY/T 33-2014) and the Nutrient Requirements of Broilers (NRC, 1994). ${ }^{3}$ DL-Met: DL-Methionine; ME: Metabolizable Energy; CP: Crude Protein.

\subsection{Data and Sampling Collection}

\subsubsection{Growth Performance}

Individual bodyweight of the birds was measured at 0 and 28 days old. Similarly, mortality and feed intake per cage were recorded daily. The growth performance of broilers was calculated by the 
average daily feed intake (ADFI), average daily body weight gain (ADG), and feed/gain ratio (FCR), which were calculated according to the data from each cage.

\subsubsection{Blood Samples}

At day 28, six birds were randomly selected from each group (1 bird from each cage) and were fasted for $12 \mathrm{~h}$ before being sacrificed. Five milliliters of blood sample per bird were collected by venipuncture of the wing vein. Serum were obtained by centrifugation at $3000 \times g$ for $10 \mathrm{~min}$ at $4{ }^{\circ} \mathrm{C}$ and stored at $-20^{\circ} \mathrm{C}$ until serum biochemical analyses. Serum biochemical indices (glucose, total protein, albumin, globulin, and uric acid) were analyzed using a BS-200 automatic blood biochemical analyzer (Mindray, Shengzhen, China).

\subsubsection{Fecal and Jejunal Mucosa Samples}

The birds were euthanized, then approximately $1 \mathrm{~g}$ of cecal feces was immediately isolated from the middle of the cecum. The cecal samples were collected into $1.5 \mathrm{~mL}$ Eppendorf tubes and quickly frozen in liquid nitrogen while being transported to the laboratory. A 5-cm segment of the intestine was cut from the midpoint of the jejunum. The tissue samples were lightly flushed with ice-cold physiological saline $(154 \mathrm{mmol} / \mathrm{L})$. Approximately $1 \mathrm{~g}$ jejunal mucosa was immediately isolated from the middle of the jejunum with sterilized surgical scissors. The jejunal mucosa samples were collected into $1.5 \mathrm{~mL}$ Eppendorf tubes and quickly frozen in liquid nitrogen while being transported to the laboratory. All of the samples were stored at $-80^{\circ} \mathrm{C}$ until further processing.

\subsubsection{Duodenum, Jejunum, and Ileum Samples}

The small intestine was promptly moved out and divided into 3 parts: the duodenum (from the pylorus to the distal point of entry of the bile ducts), jejunum (Meckel's diverticulum marked the end point of the jejunum), and ileum (the ileocecal junction marked the end of the ileum). A 1-cm segment of the intestine was cut from the midpoint of each of the duodenum, jejunum, and ileum. These intestinal tissue samples were lightly flushed with physiological saline $(154 \mathrm{mmol} / \mathrm{L})$, blotted dry with filter paper, and fixed into a $4 \%$ paraformaldehyde fixing solution at $4{ }^{\circ} \mathrm{C}$ until further analysis of intestinal mucosal morphology [20].

\subsection{Mucosal Cytokines and S-IgA}

Jejunal mucosa ( $0.5 \mathrm{~g}$ per sample) was weighted, diluted with $9 \mathrm{~mL}$ of $0.9 \%$ saline, homogenized at $4{ }^{\circ} \mathrm{C}$, and then centrifuged at $6000 \times g$ for $15 \mathrm{~min}$ at $4{ }^{\circ} \mathrm{C}$. The supernatant was harvested into $1.5 \mathrm{~mL}$ Eppendorf tubes. The concentrations of tumor necrosis factor-a (TNF- $\alpha$ ), IL-4, and secreted IgA (S-IgA) were analyzed by ELISA (Lan Bao Biological Technology Co. Ltd., Hangzhou, China).

\subsection{Measurement of Intestinal Mucosal Morphology}

One-centimeter intestinal tissue samples of the duodenum, jejunum, and ileum were embedded in paraffin. A microtome (RM-2235, Leica microsystems AG., Hessen, Germany) was used to make 5- or 6- $\mu \mathrm{m}$ slices that were mounted in glass slides and subsequently stained with hematoxylin and eosin (HE staining). Villus height (from the tip of the villus to the villus crypt junction) and crypt depth (from villus crypt junction to the base of the crypt) were measured under an Olympus Van-Ox S microscope (Opelco, Washington, DC, USA) using an image analysis software (Image-Pro, Media Cybernetics, Inc., Silver Springs, MD, USA). Six sections were taken from each intestine, and the height of the ten largest villi and the deepest crypt depth were selected for each section. From this, the villus height/crypt depth $(\mathrm{V} / \mathrm{C})$ values were calculated [20,21]. 


\subsection{Jejunum Immunohistochemistry (IHC)}

Measurement of IHC was as described previously [22]. Briefly, $1 \mathrm{~cm}$ of jejunum tissue $(4 \%$ PFA-fixed and paraffin-embedded) was cut into six sections and mounted on slides. For IHC, slides were placed in an oven with $56^{\circ} \mathrm{C}$ drying heat for $30 \mathrm{~min}$ for deparaffinization, then washed in alcohol and xylol solutions. Slides were placed in citrate buffer in a histoprocessor T/T MEGA instrument at $98^{\circ} \mathrm{C}$ for $5 \mathrm{~min}$ for antigen retrieval. Then, they were washed in phosphate-buffered saline (PBS). For blocking the endogenous peroxidase, sections were incubated in $3 \% \mathrm{H}_{2} \mathrm{O}_{2}$ for $10 \mathrm{~min}$. Slides were placed in distilled water and PBS, then incubated with primary antibody (anti-NF- $\mathrm{KB}$ pAb ab16502 Abcam, diluted, 1:50 and MAPK(ERK1/2) anti-ERK1/2 \#4696 CST, diluted, 1:1000) for $1 \mathrm{~h}$ and washed with PBS. Then, the slides were incubated with a second antibody, IgG antibody horseradish peroxidase (HRP), for $30 \mathrm{~min}$ and washed with PBS. Application of the diaminobenzidine hydrochloride chromogen for $10 \mathrm{~min}$ and washing with tap water were then performed. Only brown-colored staining was considered positive. All of slides were observed by light microscopy at $400 \times$ magnification, the selected field for counting being randomly chosen. Mean optical density of NF-KB and ERK1/2 in jejunum sections were analyzed using Image-Pro Plus 6.0 software (Media Cybernetics Inc., Bethesda, MD, USA).

\subsection{Cecal Microbiota Analysis}

DNA was extracted from cecal samples (one bird in each of the duplicate cages) using a Stool DNA Isolation Kit (Tiangen Biotech Co., Ltd., Beijing, China). The V3-V4 hypervariable region of the bacterial 16S rRNA gene were amplified by PCR, where the forward primer was 338F: $5^{\prime}$-ACTC CTACGGGAGGCAGCAG-3' ${ }^{\prime}$ and the reverse primer was 806R: $5^{\prime}$-GGAC-TACHVGGGTWTCTAAT-3' For each cecal sample, a 10-digit barcode sequence was added to the $5^{\prime}$ end of the forward and reverse primers (provided by Allwegene Company, Beijing, China). The volume of PCR reaction was $30 \mu \mathrm{L}$, containing $15 \mu \mathrm{L}$ of Phusion ${ }^{\circledR}$ High-Fidelity PCR Master Mix (New England Biolabs Inc., Beverly, MA, USA), $0.2 \mu \mathrm{M}$ of forward and reverse primers, $10 \mathrm{ng}$ of template DNA, and $13.8 \mu \mathrm{L}$ double distilled $\mathrm{H}_{2} \mathrm{O}\left(\mathrm{ddH}_{2} \mathrm{O}\right)$. Cycling parameters were $98^{\circ} \mathrm{C}$ for $1 \mathrm{~min}$, followed by 30 cycles at $98^{\circ} \mathrm{C}$ for $10 \mathrm{~s}, 57^{\circ} \mathrm{C}$ for $30 \mathrm{~s}$, and $72{ }^{\circ} \mathrm{C}$ for $30 \mathrm{~s}$, and a final extension at $72{ }^{\circ} \mathrm{C}$ for $10 \mathrm{~min}$. PCR products were mixed in equidensity ratios and purified with the GeneJET Gel Extraction Kit (Thermo Fisher Scientific Inc., Schwerte, Germany), quantified using real-time PCR, and sequenced at Allwegene Company, Beijing. The sequences were clustered into operational taxonomic units (OTUs) at a similarity level of $97 \%$ to generate rarefaction curves and to calculate the richness and diversity indices. OTUs representing $<0.005 \%$ of the population were removed and taxonomy was assigned by the Ribosomal Database Project (RDP) classifier. $\beta$-diversity was assessed by MANOVA and principal coordinate analysis. Significant differences in $\alpha$-diversity and OTU counts between the different groups were determined by one-way and two-way ANOVA analysis followed by Duncan's multiple comparison test using the SPSS (SPSS statistics 20) [23].

\subsection{Statistical Analysis}

Data were expressed as mean \pm SEM and analyzed by one-way and two-way ANOVA for single-factor and two-factor designs, respectively, using SPSS (SPSS statistics 20). In the case of the two-way analysis, interactions between treatment factors were also assessed using the SPSS program. The mean differences among different treatments were separated by Duncan's multiple range tests. A level of $p<0.05$ was used as the criterion for statistical significance. The statistical analyses used in the assessment of microbial community structure (16S rRNA sequencing) was described in Section 2.6. 


\section{Results}

\subsection{Growth Performance}

The interaction effect between E. coli challenge and ABPS on BW and ADG ( $p=0.038)$ of the broilers across the group throughout the experimental period is shown in Table 2. The ADG and final BW of birds were increased significantly with the ABPS treatment compared to the other groups, while the lowest was observed with the CNG treatment. The birds' FCR was increased in the CNG treatment and significantly different $(p<0.001)$ to the other groups. Diet supplemented with ABPS decreased FCR $(p=0.002)$. On the other hand, no significant difference was observed in the ADFI and mortality of yellow-feathered broiler chickens during E. coli challenge and ABPS treatment.

Table 2. Effects of ABPS and E. coli challenge on growth performance of yellow-feathered broilers.

\begin{tabular}{|c|c|c|c|c|c|c|c|c|}
\hline Item & \multicolumn{2}{|c|}{-E. coli } & \multicolumn{2}{|c|}{+ E. coli } & SEM & \multicolumn{3}{|c|}{$p$-Value } \\
\hline BW (g) & $635.17^{a, b}$ & $651.1^{\mathrm{a}}$ & $579.5^{c}$ & $627.33^{b}$ & 6.49 & $<0.001$ & $<0.001$ & 0.038 \\
\hline ADFI (g) & 44.83 & 44.57 & 42.58 & 44.55 & 0.39 & 0.298 & 0.168 & 0.172 \\
\hline FCR (g:g) ${ }^{1}$ & 2.08 & 2.01 & 2.17 & 2.09 & 0.02 & 0.002 & $<0.001$ & 0.658 \\
\hline
\end{tabular}

BW: body weight; ADG: average daily body weight gain; ADFI: average daily feed intake; FCR: feed/gain ratio; $\mathrm{a}, \mathrm{b}$ within a row, values with different superscripts differ significantly $(p<0.05)$; Each mean represents 6 replicates. NG: basal diet; ABP: supplemented with 500 mg ABPS additive per kg of diet; CNG: basal diet group with E. coli challenge; CABP: ABP group with E. coli challenge; ${ }^{1}$ ABPS and E. coli was significant $(p<0.05)$, and more description is provided in the results section.

\subsection{Serum Biochemistry Indices}

The results of the serum biochemical indices are shown in Table 3. There was no interaction effect between E. coli challenge and ABPS for the serum biochemical indices that were observed throughout the trial. Supplementation of ABPS increased the concentrations of total protein (TP) and globulin (GLB) $(p<0.001)$ in both E. coli-challenged and unchallenged birds. The E. coli challenge had no significant effects on GLB concentration $(p>0.05)$ compared with birds in the NG group, but had the tendency to decrease the content. Dietary ABPS supplementation with no E. coli challenge (ABP) led to decreased levels of uric acid compared with other groups, especially the NG group. At the same time, E. coli challenge resulted in an increased level of uric acid $(p<0.001)$. Serum glucose was significantly lower $(p<0.001)$ in the groups supplemented with ABPS, whether challenged with E. coli or not.

Table 3. Effects of ABPS and E. coli challenge on serum biochemical indices of yellow-feathered broilers.

\begin{tabular}{|c|c|c|c|c|c|c|c|c|}
\hline \multirow{2}{*}{ Item } & \multicolumn{2}{|c|}{$-E$. coli } & \multicolumn{2}{|c|}{$+E$. coli } & \multirow{2}{*}{ SEM } & \multicolumn{3}{|c|}{$p$-Value } \\
\hline & NG & ABP & CNG & САВР & & ABPS & E. coli & ABPS $*$ E. coli \\
\hline $\operatorname{ALB}(\mathrm{g} / \mathrm{L})$ & 13.98 & 14.66 & 13.23 & 14.18 & 0.24 & 0.104 & 0.198 & 0.763 \\
\hline $\mathrm{TP}^{1}(\mathrm{~g} / \mathrm{L})$ & 35.28 & 42.42 & 32.15 & 40.75 & 1.01 & $<0.001$ & 0.008 & 0.375 \\
\hline $\mathrm{GLB}^{2}(\mathrm{~g} / \mathrm{L})$ & 21.28 & 27.77 & 19.31 & 26.57 & 0.91 & $<0.001$ & 0.091 & 0.669 \\
\hline GLU $(\mathrm{mmol} / \mathrm{L})^{3}$ & 14.49 & 13.15 & 13.66 & 12.39 & 0.17 & $<0.001$ & $<0.001$ & 0.744 \\
\hline $\mathrm{UA}(\mu \mathrm{mol} / \mathrm{L})^{4}$ & 273.19 & 242.56 & 295.55 & 279.49 & 4.35 & $<0.001$ & $<0.001$ & 0.059 \\
\hline
\end{tabular}

GLU: glucose; TP: total protein; ALB: albumin; GLB: globulin; UA: uric acid; ${ }^{a}$ b within a row, values with different superscripts differ significantly $(p<0.05)$; Each mean represents 6 replicates. NG: basal diet; ABP: supplemented with $500 \mathrm{mg}$ ABPS additive per kg of diet; CNG: basal diet group with $E$. coli challenge; CABP: ABP group with E. coli challenge; 1,2 ABPS was significant $(p<0.05)$, and more description is provided in the results section; 3,4 ABPS and E. coli was significant $(p<0.05)$, and more description is provided in the results section.

\subsection{Immune Responses}

As shown in Figure 1, an interaction effect between E. coli challenge and ABPS was observed for IL-4 throughout the trial period. The ABP group had the lowest concentration of IL-4 in jejunal mucosa 
compared with other treatments $(p=0.04)$. Birds in E. coli-challenged groups had higher concentrations of TNF- $\alpha$ and S-IgA in jejunal mucosa than unchallenged birds $(p<0.001)$. Supplementation of ABPS increased the concentration of S-IgA in jejunal mucosa, but decreased the concentration of TNF- $\alpha$ in jejunal mucosa.
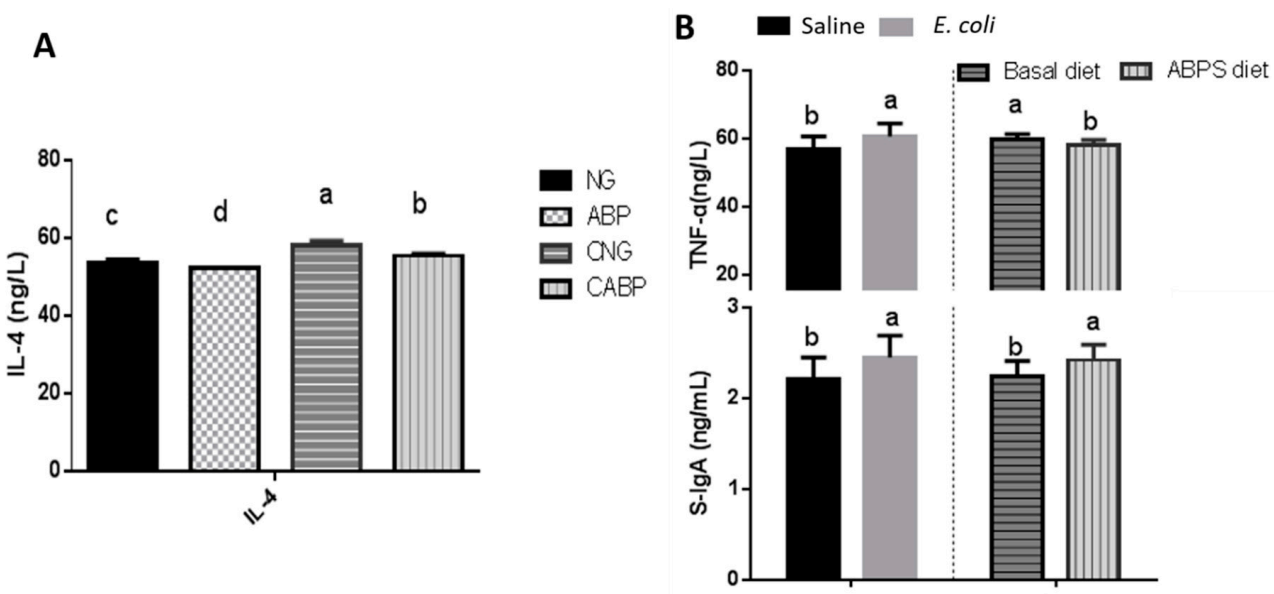

Figure 1. Effects of ABPS and E. coli challenge on mucosal cytokines and S-IgA in yellow-feathered broilers. (A) Concentration of IL-4 in jejunal mucosa; the interaction effect between $E$. coli challenge and ABPS was observed for IL-4 throughout the trial period. (B) Concentrations of TNF- $\alpha$ and S-IgA in jejunal mucosa; ABPS and E. coli treatments were significant $(p<0.05)$ for TNF- $\alpha$ and S-IgA. Values are means $\pm \mathrm{SE}, n=6$. Different superscripted lowercase letters within each group mean the values differ significantly $(p<0.05)$. NG: basal diet; ABP: supplemented with $500 \mathrm{mg}$ ABPS additive per $\mathrm{kg}$ of diet; CNG: basal diet group with E. coli challenge; CABP: ABP group with E. coli challenge.

\subsection{Response of NF- $\mathrm{B} B$ and EKR1/2 in the Jejunum}

The effects of ABPS and E. coli challenge on NF-KB and ERK1/2 in yellow-feathered broilers were detected by immunohistochemical analysis (Figure 2). The interaction between $E$. coli challenge and ABPS was observed for NF- $\mathrm{kB}$ throughout the trial. The ABP group had a lower NF- $\mathrm{kB}$ response compared to other treatments $(p<0.001)$ (Figure 2A). Birds challenged with E. coli had higher $(p<0.001)$ responses of EKR1/2 compared with the saline group. However, no significant difference $(p=0.055)$ was observed in responses of EKR1/2 in the group supplemented with ABPS (Figure 2B).

A

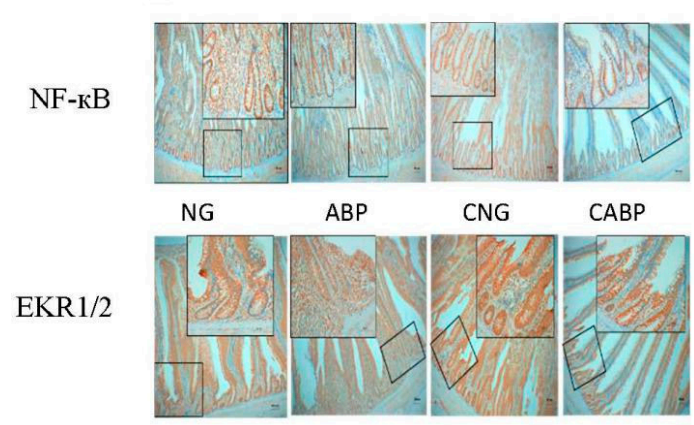

B

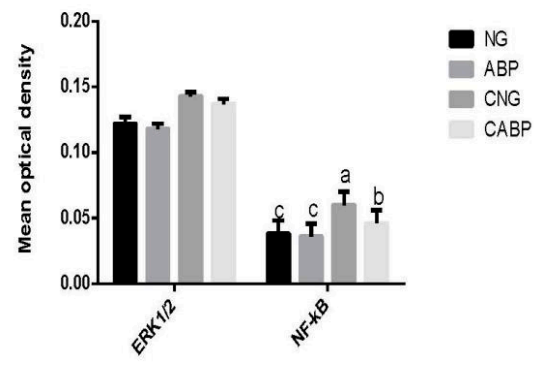

Figure 2. Response of NF- $\mathrm{B}$ B and EKR1/2 in the jejunum. (A) NF- $\mathrm{KB}$ and EKR1/2 immunostaining of mucosa (original magnification $100 \times$ and $400 \times$ ). Only brown-colored staining was considered positive. (B) Density quantification of brown-colored staining. Significant interaction between $E$. coli challenge and ABPS was observed for NF- $\mathrm{kB}$ activation, but not for ERK1/2 activation $(p=0.055)$. Values are means $\pm \mathrm{SE}, n=6$. Different superscripted lowercase letters within each group mean the values differ significantly $(p<0.05)$. NG: basal diet; ABP: supplemented with $500 \mathrm{mg}$ ABPS additive per kg of diet; CNG: basal diet group with E. coli challenge; CABP: ABP group with E. coli challenge. 


\subsection{The Change in Intestinal Mucosal Morphology}

The changes in intestinal mucosal morphologies for the duodenum, jejunum, and ileum were observed as shown in Figure 3, and the interaction effects between E. coli challenge and ABPS were observed for intestinal mucosal morphology throughout the trial. The ABP group had the highest intestinal villus height compared with other treatments $(p<0.001)$, while the CABP group had the highest villus height/crypt depth ratio $(\mathrm{V} / \mathrm{C})$ compared with other treatments $(p<0.001)$. On the other hand, the CNG group had the lowest intestinal crypt depth at the ileum compared with the other treatment groups $(p<0.001)$. The CABP group had the smallest intestinal crypt depth at the duodenum and jejunum compared with the other treatments $(p<0.001)$.
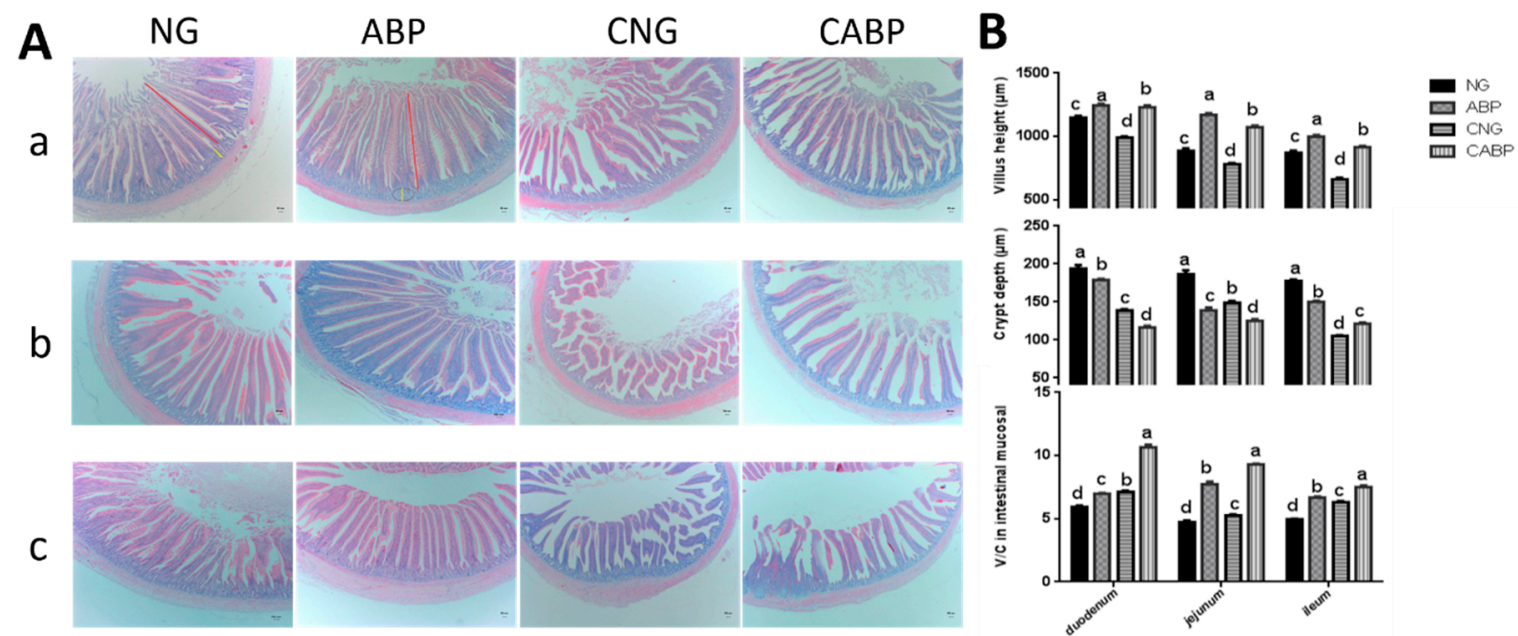

Figure 3. Effects of ABPS and E. coli challenge on intestinal mucosal morphology. (A) Intestinal (a: Duodenum; b: Jejunum; c: Ileum) mucosal morphology of 28-day-old yellow-feathered broilers was observed $(40 \times)$. (B) The villi lengths, crypt depth, and the ratio of the villus length and crypt depth $(\mathrm{V} / \mathrm{C})$ were determined in intestinal samples. Values are means $\pm \mathrm{SE}, \mathrm{n}=6$. Different superscripted lowercase letters within each group mean the values differ significantly $(p<0.05)$. NG: basal diet; ABP: supplemented with $500 \mathrm{mg}$ ABPS additive per kg of diet; CNG: basal diet group with E. coli challenge; CABP: ABP group with $E$. coli challenge.

\subsection{Cecal Microbial Community Structure}

To determine the effect of ABPS and E. coli challenge on the baseline microbial community structure, the intestinal bacterial richness and diversity was determined by $16 \mathrm{~S}$ rRNA analyses. To determine whether cecal taxa richness was altered by these treatments, we performed $\alpha$-diversity analyses using the chao1 method (Figure 4A), which showed no interaction effect of $E$. coli challenge with ABPS throughout the trial period. $\alpha$-diversity analyses demonstrated significant differences between $E$. coli-challenged and saline-receiving birds (E. coli $280.07 \pm 16.18$ vs. saline $218.89 \pm$ 16.18; $p=0.015$ ), but not between ABPS and non-ABPS birds (ABPS $266.42 \pm 16.18$ vs. non-ABPS $232.54 \pm 16.18, p=0.154)$, indicating that $E$. coli challenge reduced taxa richness in the cecal samples. Furthermore, microbial community diversity in the E. coli-challenged and non-E. coli-challenged groups was different, but not significantly ( $\beta$-diversity (unweighted Unifrac); $(p>0.05)$ ), and this was also demonstrated using principal coordinate analysis (PCoA) of the unweighted Unifrac distance matrices (Figure 4B). The PCoA plot showed that the microbial communities clustered according to challenge (E. coli-challenged vs. saline); however, there was no obvious clustering according to diet supplementation (ABPS vs. non-ABPS). The microbiota taxa composition in the cecum of birds is shown at the phylum level in Figure 4C,D and at the genus level in Table 4. As indicated, Bacteroides and Firmicutes were the dominant phyla in these samples. A significant and drastic increase in Proteobacteria and Bacteroides and a decrease in Firmicutes were observed in the E. coli-challenged groups; for example, 
Proteobacteria was more abundant in the E. coli-challenged birds (E. coli $11.92 \pm 0.004$ vs. saline, $0.63 \pm$ 0.004; $p=0.028)$, but not between ABPS and non-ABPS supplemented groups $(p>0.05)$. The interaction effect of E. coli challenge with ABPS was not observed in the Firmicutes/Bacteroidetes ratio. The E. coli challenge treatment significantly reduced the Firmicutes / Bacteroidetes ratio in birds compared with the saline treatment (E. coli $0.92 \pm 0.08$ vs. saline $1.478 \pm 0.08 ; p<0.001$ ), and also significant differences in the Firmicutes / Bacteroidetes ratio were observed between ABPS and non-ABPS birds (ABPS $1.39 \pm 0.08$ vs. non-ABPS $1.008 \pm 0.08, p=0.003$ ). In addition, there was and $E$. coli challenge $\times$ ABPS interaction effect observed for some microbial species throughout the trial period. Subsequently, E. coli-challenged birds given the ABPS-supplemented diet (group CABP) had lower E. coli and Enterococcus contents than the birds in the CNG treatment group. Also, Lachnospiraceae, Ruminococcaceae, and some genera within the Firmicutes phylum were significantly more abundant in the groups given ABPS-supplemented diets.
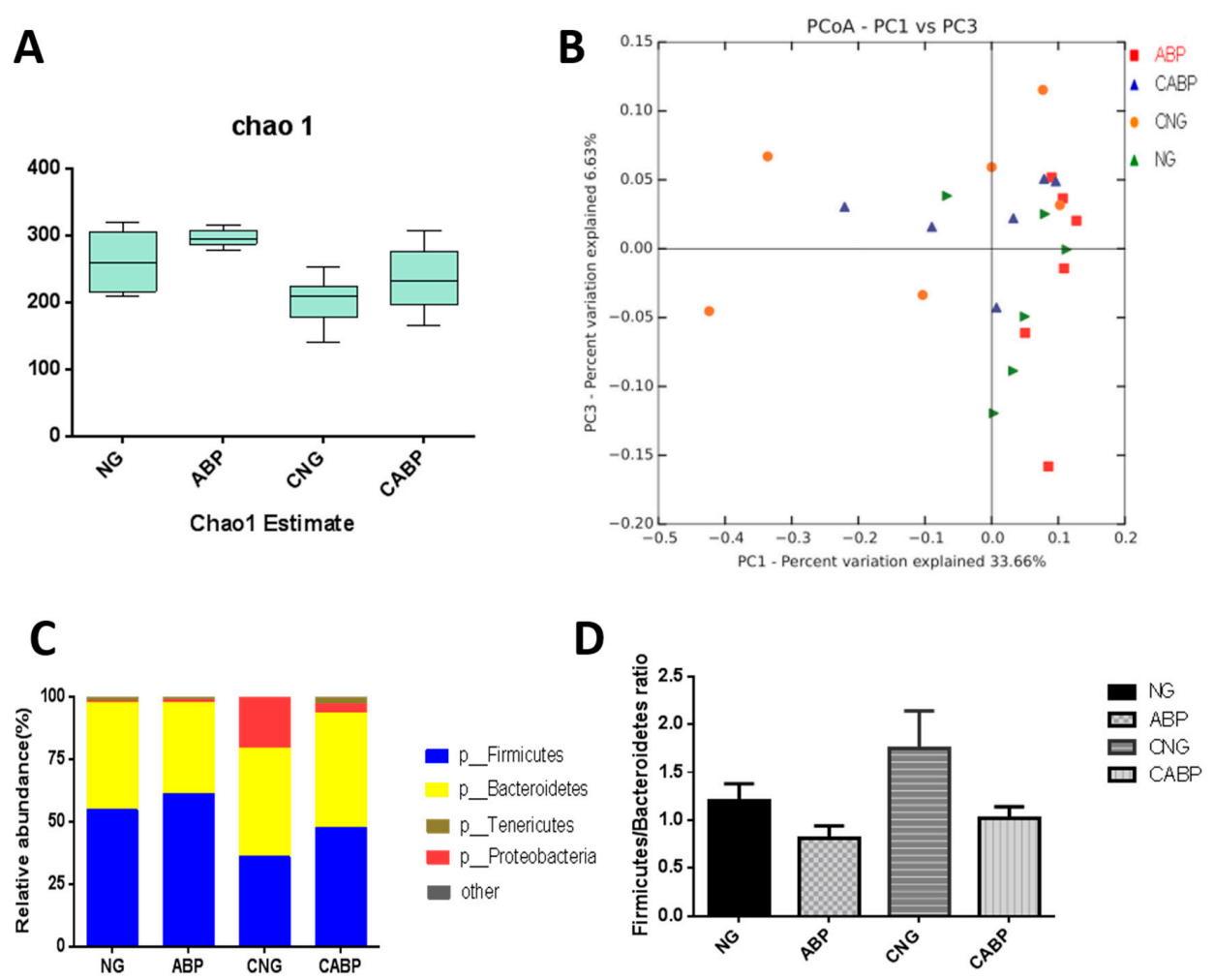

Figure 4. Effect of ABPS and E. coli challenge on the cecal microbiota. (A) Cecal taxa richness assessed by $\alpha$-diversity analyses using the chao1 method. (B) Cecal microbial community $\beta$-diversity (unweighted Unifrac, $p>0.05$ ), which was demonstrated using principal coordinate analysis (PCoA) of the unweighted Unifrac distance matrices. Microbial communities were clustered according to challenge (E. coli-challenged vs. saline); however, there was no obvious clustering according to diet supplementation (ABPS vs. non-ABPS). The first two principal coordinates (PC1 and PC3) are plotted for each bird. The percentage of dataset variability explained by each principal coordinate is shown in the axes' titles. Each dot represents one bird and each group is denoted by a different color (green, NG; orange, CNG; blue, CABP; red, ABP). (C) Microbiota taxa composition at the phylum levels is shown, labeled according to phyla (p) where available E. coli was significant $(p<0.05)$, and more description is provided in the results section. (D) The Firmicutes/Bacteroidetes ratio is shown. For the ABPS and E. coli treatment, this was significant $(p<0.05)$, and more description is provided in the results section. NG: basal diet; ABP: supplemented with $500 \mathrm{mg}$ ABPS additive per $\mathrm{kg}$ of diet; CNG: basal diet group with E. coli challenge; CABP: ABP group with E. coli challenge. 
Table 4. Effects of ABPS and E. coli challenge on some different genera in yellow-feathered broilers.

\begin{tabular}{|c|c|c|c|c|c|c|c|c|}
\hline \multirow[t]{2}{*}{ Taxonomy Units } & \multicolumn{2}{|c|}{$\begin{array}{c}-E . \text { coli } \\
\% \text { Abundance }\end{array}$} & \multicolumn{2}{|c|}{$\begin{array}{c}+ \text { E. coli } \\
\% \text { Abundance }\end{array}$} & \multicolumn{3}{|c|}{${ }^{\mathrm{ab}} p$ Value } & \multirow[t]{2}{*}{ SEM } \\
\hline & NG & $\mathbf{A B P}$ & CNG & CABP & ABPS & E. coli & ABPS $*$ E. coli & \\
\hline $\begin{array}{l}\text { p__Firmicutes; } c \_ \text {Clostridia; } o \_ \text {Clostridiales; } f \text { _Lachnospiraceae; } \\
\text { g_Coprococcus_1; s_unidentified(OTU 2) }\end{array}$ & $15.87^{a b}$ & $21.08^{a}$ & $9.31^{b}$ & $13.20^{\mathrm{b}}$ & 0.849 & 0.007 & 0.038 & 0.015 \\
\hline $\begin{array}{l}\text { p__Bacteroidetes; c_Bacteroidia; o__Bacteroidales; } \\
\text { f__Bacteroidaceae; } g \text { _Bacteroides; s_unidentified (OTU 6) }\end{array}$ & 2.13 & 3.14 & 4.31 & 4.81 & 0.056 & 0.001 & 0.474 & 0.003 \\
\hline $\begin{array}{l}\text { P_Bacteroidetes; c_Bacteroidia; o_Bacteroidales; } \\
\text { f__Bacteroidaceae; } g \_ \text {Bacteroides(OTU11) }\end{array}$ & 2.97 & 4.28 & 3.98 & 5.16 & 0.015 & 0.003 & 0.848 & 0.003 \\
\hline $\begin{array}{l}\text { p_Proteobacteria;c_Gammaproteobacteria; } \\
\text { o_Enterobacteriales;f_Enterobacteriaceae; } \\
\text { g_Escherichia-Shigella; } \text { __Escherichia_coli(OTU 4) }\end{array}$ & $0.19^{b}$ & $0.85^{b}$ & $12.34^{\mathrm{a}}$ & $3.41^{\mathrm{b}}$ & 0.055 & 0.004 & 0.031 & 0.016 \\
\hline $\begin{array}{l}\text { p__Firmicutes; } ; \text { __Bacilli; o__Lactobacillales; } f \text { _Enterococcaceae; } \\
g \text { _Enterococcus; s_unidentified(OTU 27) }\end{array}$ & $0.20^{\mathrm{b}}$ & $0.01^{\mathrm{b}}$ & $3.15^{\mathrm{a}}$ & $0.04^{\mathrm{b}}$ & 0.001 & 0.001 & 0.001 & 0.004 \\
\hline $\begin{array}{l}\text { p__Firmicutes; c_Clostridia; o_Clostridiales; } f \text { _Lachnospiraceae; } \\
\text { g_Eisenbergiella; ___unidentified(OTU 32) }\end{array}$ & 0.58 & 1.31 & 0.35 & 0.21 & 0.003 & 0.003 & 0.027 & 0.001 \\
\hline $\begin{array}{l}\text { p_Firmicutes; } c \text { _Clostridia; o_Clostridiales; } f \text { _Ruminococcaceae; } \\
\text { g__Ruminococcaceae_UCG-014; s_unidentified(OTU201) }\end{array}$ & $0.32^{b}$ & $1.17^{\mathrm{a}}$ & $0.31^{\mathrm{b}}$ & $0.34^{\mathrm{b}}$ & 0.037 & 0.005 & 0.005 & 0.001 \\
\hline $\begin{array}{l}\text { p__Firmicutes; c_Clostridia; o__Clostridiales; } \\
f \_ \text {_Ruminococcaceae(OTU 56) }\end{array}$ & 0.08 & 0.51 & 0.05 & 0.31 & 0.028 & 0.522 & 0.294 & 0.0004 \\
\hline $\begin{array}{l}\text { p_Firmicutes; c_Clostridia; o_Clostridiales; } f \text { _Ruminococcaceae; } \\
\text { g__Ruminococcaceae_UCG-014; s_unidentified(OTU63) }\end{array}$ & 0.00 & 0.43 & 0.00 & 0.11 & 0.07 & 0.189 & 0.271 & 0.001 \\
\hline
\end{tabular}

NG: basal diet; ABP: supplemented with $500 \mathrm{mg}$ ABPS additive per kg of diet; CNG: basal diet group with $E$. coli challenge; CABP: ABP group with $E$. coli challenge; ${ }^{\text {a }}$ Overall difference between groups was determined using ANOVA. ${ }^{\mathrm{b}}$ Differences between groups were determined by Duncan's multiple comparison test. ${ }^{\mathrm{a}, \mathrm{b}}$ Within a row, values with different superscripted letters differ significantly $(p<0.05)$. 


\section{Discussion}

The results of the present study indicated that E. coli challenge decreased bird's weight gain and feed intake, which is consistent with some previous studies [24,25]. Furthermore, the bird group treated with CABP had greater BW and ADG than in the CNG group, indicating the ability of ABPS to alleviate the negative effect from $E$. coli infection in birds. This observation is similar to the report of Guo et al. that ABPS supplementation had beneficial effects on the performance of weaned pigs challenged with E. coli [13]. On the other hand, serum glucose and uric acid levels were reduced in the birds fed by ABPS-supplemented diets. The glucose-lowering effect may be indicative of a role of ABPS in enhancing glucose disposal. Although the mechanisms by which ABPS decreases blood glucose concentrations are not clearly understood, the regulation of ABPS on insulin sensitivity may be involved in the response. A growing body of literature is supporting a role for ABPS supplementation in improving insulin resistance [26-28].

The present study showed that $E$. coli challenge significantly affected the birds' serum biochemical parameters such as albumin and total protein. The $E$. coli challenge reduced serum total protein levels significantly, suggesting that E. coli K88 induced immunological stress that adversely affected the health of the birds. However, these changes might be associated with the impairment of functions, such as amino acid transport and protein synthesis, usually related to oxidative stress and toxicity [29,30]. In our study, the addition of ABPS in the diet increased the levels of serum total protein, albumin, and globulin in both $E$. coli-challenged and unchallenged birds. The increased serum concentration of globulin and albumin is an indication of enhanced immune system activity, regarded as the direct reference to the body immune function [31].

In regard to the circulating concentrations of the inflammatory indices, TNF- $\alpha$ and IL- 4 were found to increase after E. coli challenge, which is consistent with the observations of Lee et al. and Liu et al. [32,33]. However, the birds fed ABPS had significantly lower concentrations of TNF- $\alpha$ and IL-4 than the birds fed the basal diet (NG) and the E. coli-challenged group (CNG), indicating that ABPS had an anti-inflammatory role. Moreover, ABPS played a significant role in suppressing the effects of $E$. coli infection in the CABP group by lowering the concentrations of TNF- $\alpha$ and IL-4 compared to the CNG group. Subsequently, the E. coli-challenged birds fed with ABPS (CABP) had the highest concentrations of S-IgA among these groups. This might be as a result of ABPS enhancing the production of S-IgA, which acts as the first line of defense against invading pathogens [34]. Many microbial pathogens make initial contact with their hosts at mucosal surfaces, especially in the alimentary tract. It has been reported that ABPS has anti-inflammatory properties and also improves body immune function, but the mechanism for its anti-inflammatory role in the animals still remains unclear $[35,36]$. NF- $\mathrm{KB}$ and MAPK signaling are important cell signaling pathways that regulate the production of cytokines and proinflammatory mediators such as IL-1, IL-6, TNF- $\alpha$, and iNOS during the inflammatory response, although the cellular signaling pathways regulating inflammation are very complicated. In this study, the birds fed with ABPS showed lower expressions of NF-KB than the birds fed either with the basal diet or receiving E. coli challenge. This result is consistent with ABPS relieving immune stress through the reduced expression levels of NF- $\mathrm{KB}$ protein in piglet jejunum epithelial cells under the immunological stress of lipopolysaccharides, as observed by Wang et al. [37].

In this study, birds fed with ABPS had longer intestinal villi compared with the other birds. The intestinal villus and crypt depth of the birds were decreased significantly under the conditions of E. coli infection in the challenge group. The challenge of E. coli K88 led to villus atrophy and intestinal morphology disruption [38]. It is known that the intact morphology of the mucosa in the duodenum, jejunum, and ileum is one of the most important indications of intestinal health as well as digestive and absorptive capacity in poultry [39,40]. Hence, our results indicated that dietary supplementation with ABPS played a beneficial role in improving the intestinal morphology of the birds, including the E. coli-challenged ones.

In this study, E. coli-challenged birds had a lower proportion of Firmicutes and an increase in the abundance of Proteobacteria. The taxonomic profiling demonstrated that ABPS treatment could 
increase the level of Firmicutes and reduce the level of Proteobacteria and Bacteroidetes, modulating the gut composition of birds to levels similar to those of the unchallenged birds. Moreover, Peter et al. and Koliada et al. found that the content of Firmicutes and the Firmicutes/Bacteroidetes ratio were gradually increased while the content of Bacteroidetes was decreased with increasing body mass index (BMI) in humans [41,42]. In line with this, our results showed that ABPS supplementation in the diet of E. coli-challenged birds could increase their BW and ADG, unlike the challenged birds with no supplement; hence, regarding the increase in the Firmicutes/Bacteroidetes ratio, a possible explanation for our findings is that Firmicutes are more effective as an energy source than Bacteroidetes, thus promoting more efficient absorption of carbohydrates and subsequent weight gain $[43,44]$. Proteobacteria is a major phylum of bacteria which includes a wide variety of pathogens, such as Escherichia, Salmonella, Vibrio, and many other notable genera [45]. In the current study, E. coli-challenged birds on the ABPS-supplemented diet had lower Escherichia coli and Enterococcus contents than the E. coli-treated birds fed a basal diet. Furthermore, in recent decades, Enterococcus faecalis has also emerged as a pathogen, especially in nosocomial infections, and this suggests that ABPS has the tendency to selectively reduce the abundance of disease-causing microorganisms [46]. The study by Xie et al. reported that ABPS increased the numbers of Lactobacilli and Bifidobacterium and reduced the numbers of $E$. coli in weaned piglets. Similarly, the present study showed that birds receiving the ABPS diet had an abundance of Ruminococcaceae and Lachnospiraceae [47]. The Ruminococcaceae, which is a producer of acetate and butyrate, is involved in the first step of microbiome-associated carbohydrate metabolism, degrading several types of polysaccharides in the intestinal tract [48]. Also, Lachnospiraceae are the producers of butyrate and have been linked to protection from colon cancer in humans $[49,50]$. The higher abundance of Ruminococcaceae and Lachnospiraceae in the ABPS-supplemented group means that short-chain fatty acid (SCFA) production was increased. Moreover, inhibition of pathogen replication has been shown to be mediated by low-molecular-weight substances, primarily SCFAs [51]. These SCFAs exert therapeutic effects on some human and experimental animal diseases $[52,53]$. The intestine harbors a complex microbial community that plays a key role in nutrition and health [54]. These results indicated that ABPS, apart from combating pathogens, may promote a more symbiotic intestinal microflora favoring the host when challenged with an enteric pathogen such as E. coli.

\section{Conclusions}

The results of the present study demonstrated that enteric disease challenge with E. coli K88 decreases growth performance in broilers, and dietary supplementation of ABPS can serve as an effective prophylactic treatment to alleviate this growth suppression. Dietary ABPS supplementation for birds could enhance the initiation of the immunological response to $E$. coli infection by mediating the release of immunoglobulins, such as S-IgA, and improving the cecal microflora. These actions finally promote the general health and growth performance of birds.

Author Contributions: Z.L. and J.H. designed the experiments. Z.L., S.O. and X.W. performed the experiments and analyzed the data. Z.L. wrote the draft manuscript; J.H., D.-X.H. and M.A.A. edited the manuscript; all other authors checked and contributed to the finalization of the manuscript.

Funding: Zhuying Liu is a student of the Joint PhD Program supported by China Scholarship Council. This project was partly supported by the National Nature Science Foundation project (grant code: 31672444 ) and the Hunan Provincial Innovation Foundation for Postgraduates (CX2017B347).

Conflicts of Interest: The authors declare no conflict of interest. 


\section{References}

1. Mead, P.S.; Slutsker, L.; Dietz, V.; McCaig, L.F.; Bresee, J.S.; Shapiro, C.; Griffin, P.M.; Tauxe, R.V. Food-Related Illness and Death in the United States. Emerg. Infect. Dis. 1999, 5, 607-625. [CrossRef] [PubMed]

2. He, C.L.; Fu, B.D.; Shen, H.Q.; Jiang, X.L.; Zhang, C.S.; Wu, S.C.; Zhu, W.; Wei, X.B. Xiang-Qi-Tang Increases Avian Pathogenic Escherichia coli-Induced Survival Rate and Regulates Serum Levels of Tumor Necrosis Factor Alpha, Interleukin-1 and Soluble Endothelial Protein C Receptor in Chicken. Biol. Pharm. Bull. 2011, 34, 379-382. [CrossRef] [PubMed]

3. Alonso, M.Z.; Padola, N.L.; Parma, A.E.; Lucchesi, P.M.A. Enteropathogenic Escherichia coli contamination at different stages of the chicken slaughtering process. Poult. Sci. 2011, 90, 2638-2641. [CrossRef] [PubMed]

4. Baurhoo, B.; Letellier, A.; Zhao, X.; Ruiz-Feria, C.A. Cecal Populations of Lactobacilli and Bifidobacteria and Escherichia coli Populations after In Vivo Escherichia coli Challenge in Birds Fed Diets with Purified Lignin or Mannanoligosaccharides. Poult. Sci. 2007, 86, 2509-2516. [CrossRef] [PubMed]

5. Zhang, L.; Cao, G.T.; Zeng, X.F.; Zhou, L.; Ferket, P.R.; Xiao, Y.P.; Chen, A.G.; Yang, C.M. Effects of Clostridium butyricum on growth performance, immune function, and cecal microflora in broiler chickens challenged with Escherichia coli K88. Poult. Sci. 2013, 93, 46-53. [CrossRef] [PubMed]

6. Mohana Devi, S.; Lee, S.I.; Kim, I.H. Effect of phytogenics on growth performance, fecal score, blood profiles, fecal noxious gas emission, digestibility, and intestinal morphology of weanling pigs challenged with Escherichia coli K88. Pol. J. Vet. Sci. 2015, 18, 557-564. [CrossRef] [PubMed]

7. Wang, Z.; Wang, L.; Chen, Z.; Ma, X.; Yang, X.; Zhang, J.; Jiang, Z. In Vitro Evaluation of Swine-Derived Lactobacillus reuteri: Probiotic Properties and Effects on Intestinal Porcine Epithelial Cells Challenged with Enterotoxigenic Escherichia coli K88. J. Microbiol. Biotechnol. 2016, 26, 1018-1025. [CrossRef] [PubMed]

8. Zhang, L.; Zhang, L.; Zhan, X.; Zeng, X.; Zhou, L.; Cao, G.; Chen, A.G.; Yang, C. Effects of dietary supplementation of probiotic, Clostridium butyricum, on growth performance, immune response, intestinal barrier function, and digestive enzyme activity in broiler chickens challenged with Escherichia coli K88. J. Anim. Sci. Biotechnol. 2016, 7. [CrossRef] [PubMed]

9. Puyalto, M.; Chamba, F.; Ortiz, A.; Torrealba, H.; Mallo, J.J.; Riboty, R. Effect of Partially Protected Sodium Butyrate on Performance, Digestive Organs, Intestinal Villi and E. coli Development in Broilers Chickens. Int. J. Poult. Sci. 2014, 13, 390-396. [CrossRef]

10. Jiang, M.-H.; Zhu, L.; Jiang, J.-G. Immunoregulatory actions of polysaccharides from Chinese herbal medicine. Expert Opin. Ther. Targets 2010, 14, 1367-1402. [CrossRef] [PubMed]

11. Vasudeva Rao, Y.; Das, B.K.; Jyotyrmayee, P.; Chakrabarti, R. Effect of Achyranthes aspera on the immunity and survival of Labeo rohita infected with Aeromonas hydrophila. Fish Shellfish Immunol. 2006, 20, $263-273$. [CrossRef] [PubMed]

12. Zou, Y.; Meng, J.; Chen, W.; Liu, J.; Li, X.; Li, W.; Lu, C.; Shan, F. Modulation of phenotypic and functional maturation of murine dendritic cells (DCs) by purified Achyranthes bidentata polysaccharide (ABP). Int. Immunopharmacol. 2011, 11, 1103-1108. [CrossRef] [PubMed]

13. Guo, G.; Liu, Y.; Fan, W.; Han, J.; Hou, Y.; Yin, Y.; Zhu, H.; Qu, Y. Effects of Achyranthes Bidentata Polysaccharide on Growth Performance, Immunological, Adrenal, and Somatotropic Responses of Weaned Pigs Challenged with Escherichia coli Lipopolysaccharide. Asian-australas. J. Anim. Sci. 2008, 21, 1189-1195. [CrossRef]

14. Kang, P.; Xiao, H.L.; Hou, Y.Q.; Ding, B.Y.; Liu, Y.L.; Zhu, H.L.; Hu, Q.Z.; Hu, Y.; Yin, Y.L. Effects of Astragalus Polysaccharides, Achyranthes bidentata Polysaccharides, and Acantbepanax senticosus Saponin on the Performance and Immunity in Weaned Pigs. Asian-Australas. J. Anim. Sci. 2010, 23, 750-756. [CrossRef]

15. Chen, Q.; Liu, Z.; He, J. Achyranthes bidentatapolysaccharide enhances immune response in weaned piglets. Immunopharmacol. Immunotoxicol. 2009, 31, 253-260. [CrossRef] [PubMed]

16. Chen, Q.; Liu, Z.; He, J.; Zhao, Y.; Wu, X. Achyranthes bidentata polysaccharide enhances growth performance and health status in weaned piglets. Food Agric. Immunol. 2011, 22, 17-29. [CrossRef]

17. Chen, Q.H.; Ding, Z.; Qiu, W.; He, J.H. Achyranthes bidentata polysaccharide decreases inflammatory cytokine secretion in weaned piglets after LPS challenge. Asian J. Anim. Vet. Adv. 2014, 13, 454-459.

18. Liu, Z.Y.; Wang, X.L.; Ou, S.Q.; Fu, C.X.; Hou, D.X.; He, J.H. Effect of Sanguinarine and Achyranthes Bidentata Polysaccharides on Performance and Immunity of Yellow Broilers. J. Poult. Sci. 2018, under review. 
19. Chen, X.M.; Xu, Y.J.; Tian, G.Y. Physical-chemical properties and structure elucidation of AbPS isolated from the root of Achyranthes bidentata. Acta Pharm. Sin. 2005, 40, 32-35. (In Chinese)

20. Xia, M.S.; Hu, C.H.; Xu, Z.R. Effects of copper-bearing montmorillonite on growth performance, digestive enzyme activities, and intestinal microflora and morphology of male broilers. Poult. Sci. 2004, 83, 1868-1875. [CrossRef] [PubMed]

21. Xu, Z.R.; Hu, C.H.; Xia, M.S.; Zhan, X.A.; Wang, M.Q. Effects of dietary fructooligosaccharide on digestive enzyme activities, intestinal microflora and morphology of male broilers. Poult. Sci. 2003, 82, 1030-1036. [CrossRef] [PubMed]

22. Agha-Hosseini, F.; Khalili, M.; Rohani, B. Immunohistochemistry Analysis of P53 and Ki-67 Proteins in Oral Lichen Planus and Normal Oral Mucosa. Iran. J. Public Health 2009, 38, 37-43.

23. Zhu, L.; Baker, S.S.; Gill, C.; Liu, W.; Alkhouri, R.; Baker, R.D.; Gill, S.R. Characterization of gut microbiomes in nonalcoholic steatohepatitis (NASH) patients: A connection between endogenous alcohol and NASH. Hepatology 2013, 57, 601-609. [CrossRef] [PubMed]

24. Cao, G.T.; Zeng, X.F.; Chen, A.G.; Zhou, L.; Zhang, L.; Xiao, Y.P.; Yang, C.M. Effects of a probiotic, Enterococcus faecium, on growth performance, intestinal morphology, immune response, and cecal microflora in broiler chickens challenged with Escherichia coli K88. Poult. Sci. 2013, 92, 2949-2955. [CrossRef] [PubMed]

25. Jing, M.; Munyaka, P.M.; Tactacan, G.B.; Rodriguez-Lecompte, J.C.; O, K.; House, J.D. Performance, serum biochemical responses, and gene expression of intestinal folate transporters of young and older laying hens in response to dietary folic acid supplementation and challenge with Escherichia coli lipopolysaccharide. Poult. Sci. 2013, 93, 122-131. [CrossRef] [PubMed]

26. Xue, S.; Chen, X.; Lu, J.; Jin, L. Protective effect of sulfated Achyranthes bidentata polysaccharides on streptozotocin-induced oxidative stress in rats. Carbohydr. Polym. 2009, 75, 415-419. [CrossRef]

27. Yang, X.D.; Zhang, J.; Shen, M.S. Effect of Achyranthes Bidentata Polysaccharides on Expression of Adipose Differentiation-Related Protein Gene in Type 2 Diabetes Mellitus Rats. Pharm. Biotechnol. 2010, 17, 334-336. (In Chinese)

28. He, K.; Li, X.; Chen, X.; Ye, X.; Huang, J.; Jin, Y.; Li, P.; Shu, H. Evaluation of antidiabetic potential of selected traditional Chinese medicines in STZ-induced diabetic mice. J. Ethnopharmacol. 2011, 137, 1135-1142. [CrossRef] [PubMed]

29. Hassan, H.M.; Fridovich, I. Superoxide radical and the oxygen enhancement of the toxicity of paraquat in Escherichia coli. J. Biol. Chem. 1978, 253, 8143-8148. [PubMed]

30. Cuevas-Ramos, G.; Petit, C.R.; Marcq, I.; Boury, M.; Oswald, E.; Nougayrede, J.-P. Escherichia coli induces DNA damage in vivo and triggers genomic instability in mammalian cells. Proc. Natl. Acad. Sci. USA 2010, 107, 11537-11542. [CrossRef] [PubMed]

31. Zhou, H.; Zhao, Y.; Liu, X. Path analysis of the relationship between serum protein contents and the growth traits in chicks. Acta Vet. Zootech. Sin. 1994, 25, 301-305. (In Chinese)

32. Lee, D.N.; Shen, T.F.; Yen, H.T.; Weng, C.F.; Chen, B.J. Effects of Chromium Supplementation and Lipopolysaccharide Injection on the Immune Responses of Weanling Pigs. Asian-Australas. J. Anim. Sci. 2000, 13, 1414-1421. [CrossRef]

33. Liu, Y.; Huang, J.; Hou, Y.; Zhu, H.; Zhao, S.; Ding, B.; Yin, Y.; Yi, G.; Shi, J.; Fan, W. Dietary arginine supplementation alleviates intestinal mucosal disruption induced by Escherichia coli lipopolysaccharide in weaned pigs. Br. J. Nutr. 2008, 100, 552-560. [CrossRef] [PubMed]

34. Muir, W.I.; Bryden, W.L.; Husband, A.J. Evaluation of the efficacy of intraperitoneal immunization in reducing Salmonella typhimurium infection in chickens. Poult. Sci. 1998, 77, 1874-1883. [CrossRef] [PubMed]

35. Zhu, X.; Pan, Y.; Zheng, L.; Cui, L.; Cao, Y. Polysaccharides from the Chinese medicinal herb Achyranthes bidentata enhance anti-malarial immunity during Plasmodium yoelii 17XL infection in mice. Malar. J. 2012, 11, 49. [CrossRef] [PubMed]

36. Ou, N.; Sun, Y.; Zhou, S.; Gu, P.; Liu, Z.; Bo, R.; Hu, Y.; Liu, J.; Wang, D. Evaluation of optimum conditions for Achyranthes bidentata polysaccharides encapsulated in cubosomes and immunological activity in vitro. Int. J. Biol. Macromol. 2018, 109, 748-760. [CrossRef] [PubMed]

37. Wang, X.; Li, M.W.; Ma, J.; Chen, Q.H. Achyranthes bidentata Polysaccharides:Regulation on Immunological Stress in Piglet Intestinal Epithelial Cells and Its Action Mechanism. Chin. J. Anim. Sci. 2017, 29, 4116-4122. 
38. Yi, G.F.; Carroll, J.A.; Allee, G.L.; Gaines, A.M.; Kendall, D.C.; Usry, J.L.; Toride, Y.; Izuru, S. Effect of glutamine and spray-dried plasma on growth performance, small intestinal morphology, and immune responses of Escherichia coli K88+-challenged weaned pigs. J. Anim. Sci. 2005, 83, 634-643. [CrossRef] [PubMed]

39. Olnood, C.G.; Beski, S.S.M.; Iji, P.A.; Choct, M. Delivery routes for probiotics: Effects on broiler performance, intestinal morphology and gut microflora. Anim Nutr. 2015, 1, 192-202. [CrossRef] [PubMed]

40. Roofchaei, A.; Rezaeipour, V.; Vatandour, S.; Zaefarian, F. Influence of dietary carbohydrases, individually or in combination with phytase or an acidifier, on performance, gut morphology and microbial population in broiler chickens fed a wheat-based diet. Anim. Nutr. 2017. [CrossRef]

41. Turnbaugh, P.J.; Bäckhed, F.; Fulton, L.; Gordon, J.I. Diet-Induced Obesity Is Linked to Marked but Reversible Alterations in the Mouse Distal Gut Microbiome. Cell Host Microbe 2008, 3, 213-223. [CrossRef] [PubMed]

42. Koliada, A.; Syzenko, G.; Moseiko, V.; Budovska, L.; Puchkov, K.; Perederiy, V.; Gavalko, Y.; Vaiserman, A. Association between body mass index and Firmicutes/Bacteroidetes ratio in an adult Ukrainian population. BMC Microbiol. 2017, 17. [CrossRef] [PubMed]

43. Turnbaugh, P.J.; Ley, R.E.; Mahowald, M.A.; Magrini, V.; Mardis, E.R.; Gordon, J.I. An obesity-associated gut microbiome with increased capacity for energy harvest. Nature 2006, 444, 1027-1031. [CrossRef] [PubMed]

44. Krajmalnik-Brown, R.; Ilhan, Z.E.; Kang, D.W.; DiBaise, J. K Effects of Gut Microbes on Nutrient Absorption and Energy Regulation. Nutr. Clin. Pract. 2012, 27, 201-214. [CrossRef] [PubMed]

45. Madigan, T.M.; Martinko, J.M. Brock Biology of Microorganisms, 11th ed.; Prentice Hall: Upper Saddle River, NJ, USA, 2005; pp. 122-185, ISBN 0-13-144329-1.

46. Fisher, K.; Phillips, C. The ecology, epidemiology and virulence of Enterococcus. Microbiology 2009, 155, 1749-1757. [CrossRef] [PubMed]

47. Xie, H.B.; Zou, Y.; Liu, L.L.; Yang, Y.S.; He, J.H. Effects of Botanical Polysaccharide on Growth Performance and Intestinal Environment of Weaned Piglets. Chin. J. Anim. Nutr. 2018, 30, 2662-2671.

48. Flint, H.J.; Bayer, E.A.; Rincon, M.T.; Lamed, R.; White, B.A. Polysaccharide utilization by gut bacteria: Potential for new insights from genomic analysis. Nat. Rev. Microbiol. 2008, 6, 121-131. [CrossRef] [PubMed]

49. Louis, P.; Young, P.; Holtrop, G.; Flint, H.J. Diversity of human colonic butyrate-producing bacteria revealed by analysis of the butyryl-CoA:acetate CoA-transferase gene. Environ. Microbiol. 2010, 12, 304-314. [CrossRef] [PubMed]

50. Meehan, C.J.; Beiko, R.G. A Phylogenomic View of Ecological Specialization in the Lachnospiraceae, a Family of Digestive Tract-Associated Bacteria. Genome Biol. Evol. 2014, 6, 703-713. [CrossRef] [PubMed]

51. Oelschlaeger, T.A. Mechanisms of probiotic action-A review. Int. J. Med. Microbiol. 2010, 300, 57-62. [CrossRef] [PubMed]

52. Jung, T.H.; Park, J.H.; Jeon, W.M.; Han, K.S. Butyrate modulates bacterial adherence on LS174T human colorectal cells by stimulating mucin secretion and MAPK signaling pathway. Nutr. Res. Pract. 2005, 9, 343. [CrossRef] [PubMed]

53. Macfarlane, G.T.; Macfarlane, S. Bacteria, Colonic Fermentation, and Gastrointestinal Health. J. AOAC Int. 2012, 95, 50-60. [CrossRef] [PubMed]

54. Kong, Q.; He, G.Q.; Jia, J.L.; Zhu, Q.L.; Ruan, H. Oral Administration of Clostridium butyricum for Modulating Gastrointestinal Microflora in Mice. Curr. Microbiol. 2010, 62, 512-517. [CrossRef] [PubMed]

(C) 2018 by the authors. Licensee MDPI, Basel, Switzerland. This article is an open access article distributed under the terms and conditions of the Creative Commons Attribution (CC BY) license (http://creativecommons.org/licenses/by/4.0/). 